\title{
Molecular Biology... What Molecular (systems) Biology?
}

\section{Miguel Ángel Medina*}

Department of Molecular and Biochemistry, University of Málaga, Málaga, Spain

The launch of a new Molecular Biology journal can induce questions as: Is it necessary a new Molecular Biology journal? What kind of Molecular Biology journal? The overwhelming number of molecular biology articles currently published and the high number of molecular biology journals currently available are both a reality and a sign of the relevance the discipline Biochemistry and Molecular Biology has achieved in the scientific world. Undoubtedly, Molecular Biology has played an essential role in the great scientific-technological revolution of recombinant DNA, which has allowed in less than thirty years to push Biology up to its privileged current position as the leading science at the forefront or XXI ${ }^{\text {st }}$ Century research. The enormous success of Molecular Biology and DNA recombinant technology, along with the emergence of Bioinformatics allowed for the launch of a full battery of genome projects in the nineties leaded by the Human Genome Project. Its goals were reached 15 years in advance of previsions thanks to a big step ahead of available molecular biology technologies allowing for the first time in Biology a true high throughput and high content analysis of data. Post-genomic Molecular Biology has yielded the emergence of so many "omics" (proteomics, transcriptomics, interactomics, and metabolomics, among others) as to warrant the validity of the motto "Put an omics in your (scientific) life". At the beginning of our current century it began to appear clear that Molecular Biology could "die" because of its enormous success. On the one hand, the great efficacy and productivity of the reductionist approach of pre- and post-genomic Molecular Biology has yielded an overwhelming accumulation of detailed biological data at molecular level. This has occurred at more and more accelerated rates, leading to an accumulation of new data at higher rates than those required for their processing to transform these data in new information and finally in actual new knowledge. On the other hand, this enormous advance in detailed molecular knowledge has not been accompanied by an equivalent advance in our degree of understanding of functional processes in living beings. Reductionism is based on the Cartesian principles of analysis and synthesis, according to which complex problems can be divided into an array of simpler problems to be autonomously analyzed, with the assumption that the summation of partial solutions could give account for the solution of the whole problem. Reductionism also assumes the Occam's razor principle, according to which when there are several alternative possible solutions the simplest one has the highest probability to be the true one. However, neither the Cartesian principles nor the Occam's razor principle can always be applied to complex biological systems. In their influential end of millennium essay "From Molecular to Modular Cell Biology", Hartwell et al. [1] envisioned a needed change within the hard core of reductionist molecular biology towards "new" systemic, holistic approaches able to assume that emergence pervades all biological systems:

"Much of twentieth-century biology has been an attempt to reduce biological phenomena to the behaviour of molecules. (...) In contrast, most biological functions arise form interactions among many components. (...) We argue here for the recognition of functional "modules" as a critical level of biological organization. (...) We believe that general "design principles" - profoundly shaped by the constraints of evolutiongovern the structure and function of modules. Finally, the notion of function and functional properties separates Biology from other natural sciences and links it to synthetic disciplines such as computer sciences and engineering" [1].
In my opinion, there have not been yet the proper acknowledge to the influence of this essay in the powerful emergence of two new biological disciplines in these initial years of the current century, namely, Systems Biology and Synthetic Biology. Although some (or many) scientists understand Systems Biology as a collection of tools allowing for the management of the huge amount of new biological data obtained by "omics" approaches and available in biological data bases, Systems Biology appeared with the purpose of providing new insights concerning the complexity of biological process. In this point, it could be advisable to remind that in December 1999 Maurice Wilkins (then, the Editor-in-Chief of the journal BioEssays) wrote an Editorial pointing to Complexity as one of the three main challenges -along with Consilience and Communication- for the Biology of XXI ${ }^{\text {st }}$ Century [2]. Here it is also advisable to acknowledge that genuine systemic approaches to the study of biological issues can be traced back along the whole history of Biology, well before this "new" Systems Biology comes of age.

In 2002, the first published overview of the then nascent "new" Systems Biology [3] established that the study of any biological system as a whole would require: 1 . The knowledge of the structure of this system. 2. The understanding of the dynamics of the system. 3. The identification of the design principles justifying both the structure and the dynamics of the system. 4. The identification of the rules of control and regulation governing the behaviour of the system. In the roughly ten years that have gone by since then, Systems Biology has developed mainly in a kind of Molecular Systems Biology, as exemplified by the successful publication of Nature editorial group with this name. In fact, according to Leroy Hood (President of the Institute for Systems Biology -created in 2000 as the first worldwide multidisciplinary scientific centre devoted to Systems Biology- at Seatle, USA): "Systems Biology is the science of discovering, modeling, understanding and ultimately engineering at the molecular level the dynamic relationships between the biological molecules that define living organisms". According to Westerhoff et al. [4], Systems Biology "aims to address the molecular biology basis of biological function". Thus, Molecular Biology should not be understood as in opposition to but rather as complementary to "classical" and high-content Molecular Biology approaches. What's next? According to Chuang et al. [5], four emerging applications of Systems Biology for this decade are: 1. Pathway-based biomarker identification. 2. Global genetic interaction network analysis. 3. Stem cell systems biology. 4. Systems approaches to identify disease genes. In relation to this last biomedical application, it seems clear that the disease-ome and its identification is a key goal [6]. Other future useful applications of Molecular Systems Biology to Medicine will include

*Corresponding author: Miguel Ángel Medina Department of Molecular and Biochemistry, University of Málaga, Málaga, Spain and CIBER de Enfermedades Raras (CIBERER), Málaga, Spain, Tel: +34-952137132; Fax: +34-952132000 E-mail:medina@uma.es

Received January 04, 2012; Accepted January 06, 2012; Published January 08 2012

Citation: Medina MÁ (2012) Molecular Biology... What Molecular (systems) Biology? Mol Biol 1:e101. doi:10.4172/2168-9547.1000e101

Copyright: @ 2012 Medina MÁ. This is an open-access article distributed under the terms of the Creative Commons Attribution License, which permits unrestricted use, distribution, and reproduction in any medium, provided the original author and source are credited. 
drug discovery and development, behavioral influences on disease propensity, and metagenomics [6].

In the other extreme of the conceptual axis reductionism/holism, the emergence of single molecule detection and analysis techniques has allowed scientists for the first time to have access to the study of molecules at the individual level, one by one, representing the most extreme reductionist approach to the study of biological systems.

All these approaches, from "classical" Molecular Biology to "omics", from Systems Biology to Single Molecule Analysis should have and will have their place in this new Molecular Biology journal that with its open access format warrants the rapid dissemination and universal accessibility of the significant contributions within this expanded (and expanding) field expected to be published here. We are waiting for your contributions: join us!

\section{Acknowledgements}

The experimental work in my research group is supported by grants
PS09/02216 (Spanish Ministry of Science and Innovation, ISCIII and FEDER), and PIE P08-CTS-3759, CVI-6585 and funds from group BIO-267 (Andalusian Government and FEDER). The "CIBER de Enfermedades Raras" is an initiative from the ISCIII (Spain).

\section{References}

1. Hartwell LH, Hopfield JJ, Leibler S, Murray AW (1999) From molecular to modular cell biology. Nature 402: C47-C52.

2. Wilkins M (1999) Consilience, complexity and communication: three challenges at the start of the new century. BioEssays 21: 983-984.

3. Kitano H (2002) Systems Biology: a brief overview. Science 295: 1662-1664.

4. Westerhoff HV, Winder C, Messiha H, Simeonidis E, Adamczyk M, et al. (2009) Systems biology: the elements and principles of life. FEBS Lett 583: 38823890

5. Chuang HY, Hofree M, Ideker T (2010) A decade of systems biology. Annu Rev Cell Dev Biol 26: 721-744.

6. Loscalzo J, Barabasi AL (2011) Systems biology and the future of medicine. WIREs Syst Biol Med 3: 619-627. 